\title{
Designing a data structure for polyhedral surfaces
}

\section{Report}

Author(s):

Kettner, Lutz

Publication date:

1997

Permanent link:

https://doi.org/10.3929/ethz-a-006652205

Rights / license:

In Copyright - Non-Commercial Use Permitted

Originally published in:

Technical Report / ETH Zurich, Department of Computer Science 278 


\title{
Designing a Data Structure for Polyhedral Surfaces
}

\author{
Lutz Kettner* \\ Institut für Theoretische Informatik, ETH Zürich, CH-8092 Zürich, Switzerland
}

December 8, 1997

\begin{abstract}
Design solutions for a program library are presented for combinatorial data structures in computational geometry, such as planar maps and polyhedral surfaces. Design issues considered are genericity, flexibility, time and space efficiency, and ease-of-use. We focus on topological aspects of polyhedral surfaces. Edgebased representations for polyhedrons are evaluated with respect to the design goals. A design for polyhedral surfaces in a halfedge data structure is developed following the generic programming paradigm known from the Standard Template Library STL for C++. Connections are shown to planar maps and face-based structures managing holes in facets.
\end{abstract}

Author's address:

Institute for Theoretical Computer Science

ETH Zentrum

CH-8092 Zurich

Switzerland

email: kettner@inf.ethz.ch

Technical Report \#278, December 1997

Departement Informatik, ETH Zürich.

Electronically available from:

ftp://ftp.inf.ethz.ch/pub/publications/tech-reports/

${ }^{*}$ Part of this research was carried out while the author was at the Freie Universität Berlin with a scholarship from the Graduiertenkolleg "Algorithmische Diskrete Mathematik", DFG We 1265/2-1. Support from the ESPRIT IV LTR Project No. 21957 (CGAL) is also acknowledged. 


\section{Introduction}

Combinatorial structures, such as planar maps, are fundamental in computational geometry. In order to use computational geometry in practice, a solid library must provide generic and flexible solutions as one of its fundamental cornerstones. Other design criteria are time and space efficiency. Ease-of-use is necessary to make the power of a design accessible and to attract users. We report a solution proposed for the Computational Geometry Algorithms Library $\mathrm{CGAL}^{1}$, which is a joint effort of seven academic institutes in Europe [8, 25].

We focus on edge-based representations of three-dimensional polyhedral surfaces and illustrate connections to planar maps and face-based structures which may have holes in their facets. We concentrate on the topological aspects and derive solutions applicable to other data structures as well. In particular, we want to vary the internal storage organization and the kind of incidences that are actually stored. Additional user data can be integrated easily. A top-level interface ensures ease-of-use and combinatorial integrity. On the other hand, a protected access to the internal representation is granted.

In the first part of the paper we define polyhedral surfaces and review known edgebased boundary representations. In the second part we start with a short introduction to the modern design principles available in $\mathrm{C}^{++}$and known as the generic programming paradigm from the Standard Template Library, STL [29, 23, 4, 27]. We derive design goals and evaluate previous work. We continue with an overview of our design, present several aspects in more detail and conclude with its evaluation. The two main advantages of our design are: The flexibility is completely handled at compile time, i.e. there is no runtime overhead due to the flexibility, and memory is only allocated for the features actually used. For example, a polyhedron with no information in facets does not allocate facet nodes and facet pointers at all. The appendix gives a short introduction to $\mathrm{C}^{++}$novices how to read the $\mathrm{C}++$ example programs and several examples from the soon-to-be-available CGAL Release 1.0.

\section{Polyhedral Surfaces}

A boundary representation of a polyhedral surface consists of a set of vertices $V$, a set of edges $E$, a set of facets $F$ and an incidence relation on them. For a living example see Figure 1.

The two types of boundary representations are 2-manifold and non-manifold surfaces. A 2-manifold surface is a surface where for each point on the surface there exists a neighborhood that is homeomorphic to the open disc [19, 13]. Non-manifold examples

\footnotetext{
${ }^{1}$ http://www.cs.ruu.nl/CGAL/
} 


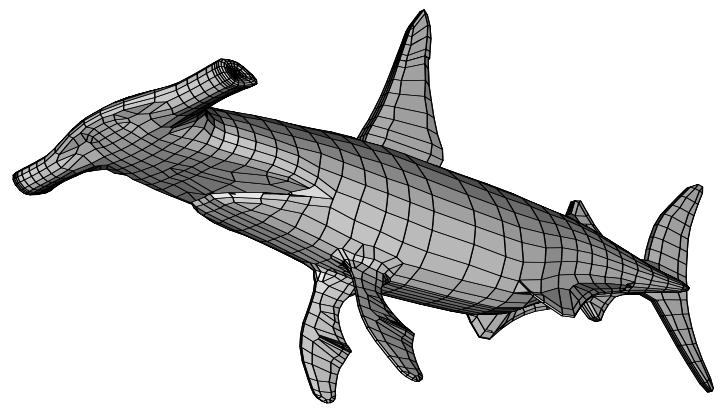

Figure 1: Hammerhead, an orientable 2-manifold of 2560 vertices. This one is homeomorphic to a sphere.
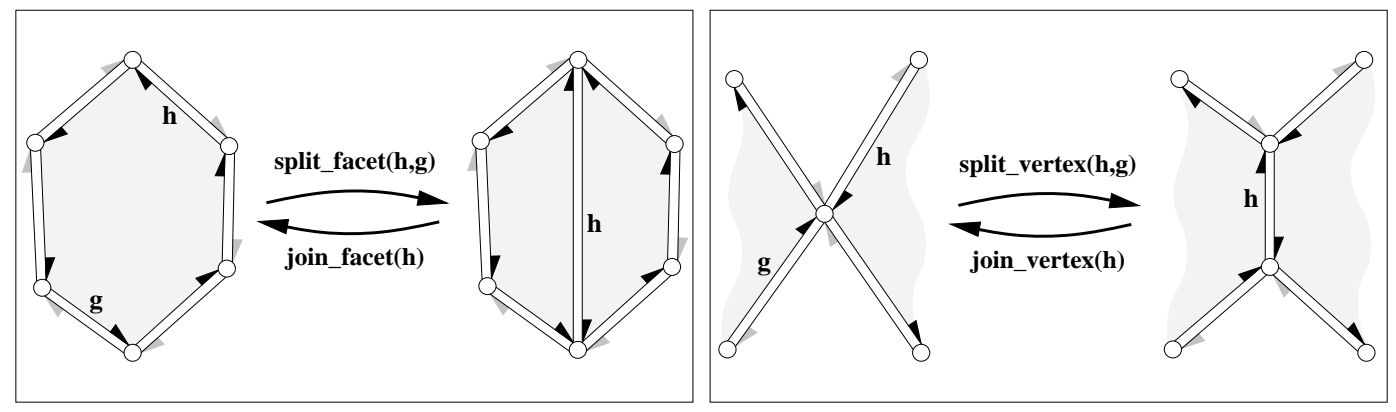

Figure 2: Euler operator examples for polyhedral surfaces.

are two tetrahedrons glued together at a single vertex or a common edge. The next distinction is between orientable and non-orientable 2-manifold surfaces. Without going into details, a surface is orientable if a consistent orientation can be assigned to each facet such that for each edge the two incident facets have opposite orientations at this edge. An example of a non-orientable 2-manifold is the Klein bottle. We consider only orientable 2-manifolds.

The natural operations under which 2-manifolds are closed are Euler operations; Four of them are shown in Figure 2. The principal characteristic of an Euler operation is the invariance of the Euler-Poincaré formula. A sufficiency proof for a specific set of Euler operations can be found in [19]. Note that 2-manifolds are not closed under (regularized) boolean operations.

The class of representable surfaces is further restricted by the kind of geometry associated with vertices, edges and faces. Vertices map to points in $\mathbb{R}^{3}$. For polyhedra the edges are typically the straight line segments between their two endpoints and the facets are simple, planar polygons. Other classes might allow curved surfaces as facets.

We now present a definition for polyhedral surfaces following Steinitz [28]. It is the basis for the combinatorial integrity definition of the polyhedral surface data structure 
and will lead to a stricter class of representable surfaces, which provides more insight in the combinatorial structure of the representation.

Definition 2.1. A structural complex is a union $C=V \cup E \cup F$ of three disjoint sets together with an incidence relation. We call $V$ the vertices, $E$ the edges and $F$ the facets of the structural complex. The incidence relation on $C$ must be symmetric. No two elements from the same set $V, E$ or $F$ are incident. If $v \in V$ is incident to $e \in E$ and $e$ is incident to $f \in F$ then $v$ is incident to $f$.

Definition 2.2. A polyhedral complex is a structural complex with four additional conditions.

(1) Every edge is incident to two vertices.

(2) Every edge is incident to two facets.

(3) For every incident pair $v, f$, there are exactly two edges incident to both.

(4) Every vertex and every facet is incident to at least one other element.

The neighborhood of a vertex is the set of edges and facets incident to the vertex. If we restrict the incidence relation to this neighborhood then each facet is incident to exactly two edges and each edge is incident to exactly two facets. The neighborhood decomposes into disjoint cycles. As for the dual, the neighborhood of a facet is the set of incident edges and vertices and decomposes into cycles too. Assuming that the neighborhood of each facet is a single cycle (geometrically speaking: no holes in the facet), we can define a polyhedral complex as oriented if each cycle around a facet is oriented and if, for each edge, the two cycles of its two incident facets are oriented in opposite directions. A polyhedral complex is orientable if there exists such an orientation.

Definition 2.3. The boundary representation of a polyhedron is a polyhedral complex with a mapping $V \rightarrow \mathbb{R}^{3}$. This extends to the edges by mapping them to the open, straight line segments between their two incident endpoints. The following additional conditions must hold.

(5) The neighborhood of each vertex and each facet is a single cycle.

(6) The polyhedral complex is orientable.

(7) The mapping of the cycle of the neighborhood of each facet is the boundary of a simple, planar polygon. The mapping extends for $F$ to the open region of these polygons. 
(8) The images of $V, E$ and $F$ are pairwise disjoint.

The surface defined by such a boundary representation is an orientable 2-manifold where the neighborhoods of two vertices have at most one edge and two facets in common, the edge and vertex graphs are connected within each connected component of the surface and where each facet has at least three edges on its boundary. The smallest possible configuration is a tetrahedron.

The closed surfaces considered so far can be extended to surfaces with boundaries by two changes in the definition: Condition (2) can be relaxed to allow edges that are incident to one facet; they are called border edges. This induces a modification of (5): The neighborhood of a vertex decomposes into either a cycle or a collection of open paths going from border edge to border edge. Although the surface is no longer closed, the orientation still defines a "solid" side of the surface. The minimal configuration for surfaces with boundaries is a triangle. The data structures we will describe can be used for polyhedrons as well as for surfaces with boundaries with a simple extension denoting "empty" facets.

A suitable data structure based on the Definition 2.3 for polyhedral surfaces has been used successfully for three years in a project on contour-edge-based polyhedron visualization where we take advantage of the strict properties imposed by the definitions: For example the definition for contour-edges is based on the orientable 2-manifold property, and the lack of holes in facets simplifies certain algorithms ${ }^{2}$ [16]. An initial implementation of the data structure made it easy to compute the silhouette for a polyhedral surface [14]. The extension of this data structure design and their advantages are presented in the following sections.

\section{Data Structures for Boundary Representations}

The following survey of edge-based data structures addresses their sufficiency for modeling topology and the efficiency of their primitive operations and storage costs. The representative example chosen is the traversal around a vertex to the next counterclockwise edge.

\footnotetext{
${ }^{2}$ And holes are not represented in the file-formats that occur usually in visualization, for example VRML [2, 31], Open Inventor [33] or the Object File Format OFF [26]. These consist of a list of vertices followed by a list of facets. Each facet is a list of indices denoting a subset of the points. Edges are not explicitly stored but can be derived from the vertices shared by facets. These formats are not strict enough for our purpose since they can represent non-manifold configurations where three or more facets are incident to a single edge, non-orientable 2-manifolds, and also violate condition (3) for polyhedral complexes. But they cannot represent holes in facets.
} 


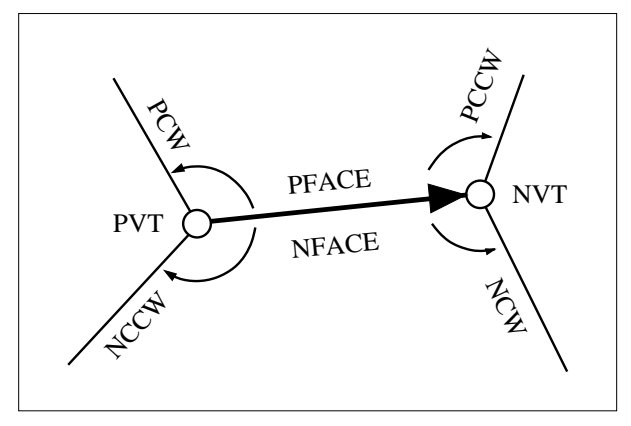

Figure 3: A winged-edge.

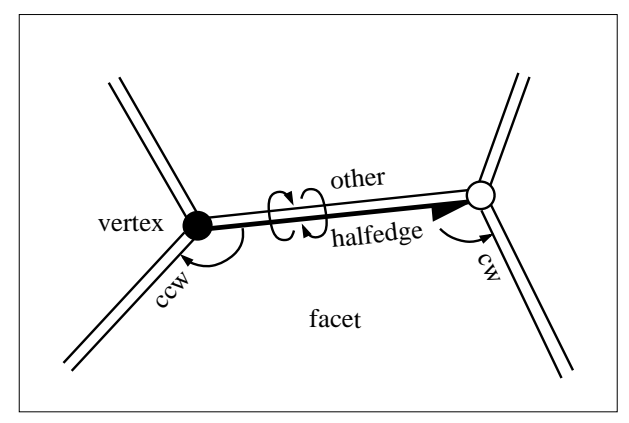

Figure 4: FE-structure.

Winged-Edge Data Structure. The winged-edge data structure $[1,11]$ stores, for each oriented edge, eight references: two vertices (PVT, NVT), two faces (PFACE, NFACE) and four incident edges that share the same faces and vertices (PCW, PCCW, NCW and NCCW), the so-called wings, see Figure 3. An edge is oriented from the source vertex PVT to the target vertex NVT. The face PFACE is to the left of the oriented edge when the surface is seen from the outside.

This data structure is able to model orientable 2-manifolds. It is even sufficient for curved-surface environments where loops and multi-edges are allowed [32]. The basic operations include traversal around a vertex and around a facet. High-level operations maintaining integrity are Euler operators. The next edge counterclockwise around a vertex $\mathrm{v}$ for an edge $\mathrm{e}$ is equal to $\mathrm{e}->\mathrm{PCW}$ if $\mathrm{e}->\mathrm{PVT}==\mathrm{v}$ and $\mathrm{e}->\mathrm{NCW}$ otherwise.

Variants are possible where vertex and facet pointers can even be omitted without losing the traversal capabilities knowing the edge visited previously. However, all four edge pointers must remain if loops or multi-edges are allowed since otherwise the traversal around a vertex or facet is no longer uniquely defined [32]. The winged-edge data structure where the wings PCCW and NCCW are omitted has been called Doubly Connected Edge List (DCEL) by [22] though this name is now more commonly used for the halfedge data structure [7]. ${ }^{3}$

The two symmetric parts in the winged-edge correspond to the two possible orientations of the edge. The inefficient case distinction in the traversal computation results from the fact that a pointer to an edge does not encode the orientation it is currently used with. One extension of the winged-edge maintains an additional bit with each edge-pointer to code the orientation, but this leads to cumbersome storage layouts and function interfaces.

Halfedge Data Structure. The orientation problem in the winged-edge data structure can be solved by splitting the edge into the two symmetric records, called halfedges,

\footnotetext{
${ }^{3}$ In order to avoid confusion we will not use the name DCEL since it turned out to be ambiguous. In fact, the name is misleading when denoting halfedges and the possible variants of single linking.
} 


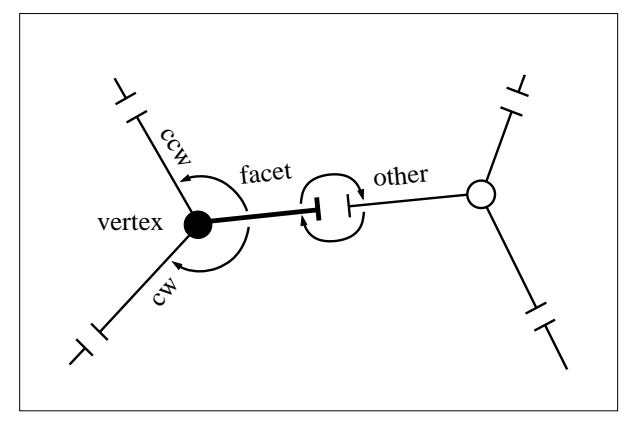

Figure 5: VE-structure.

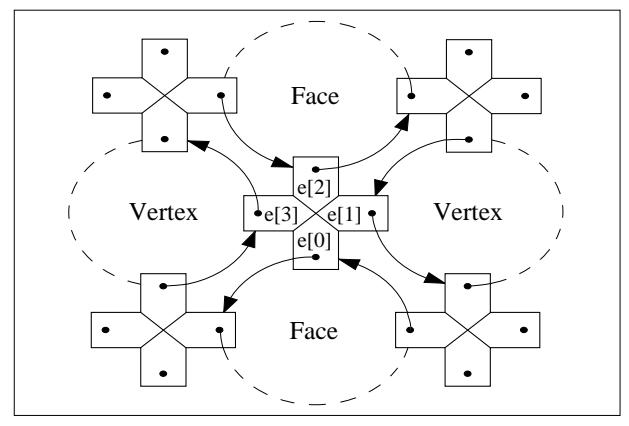

Figure 6: Quad-edge data structure.

and adding mutual links to each other [32,3]. There are two ways of splitting the edge, which are actually dual to each other. In both situations the halfedge contains a pointer to an incident vertex, an incident facet and the opposite halfedge. It is a matter of convention whether the source or target vertex is the one chosen to be stored in a halfedge or whether the facet to the left or the right is stored. In [32] the source vertex and the facet to the right were chosen. The FE-structure in Figure 4 additionally stores a pointer to the next clockwise halfedge and optionally a pointer to the previous counterclockwise halfedge around the facet. It is therefore biased towards traversals around the incident facet. The dual VE-structure is depicted in Figure 5. Its next and optional previous pointer refer to halfedges counterclockwise and clockwise around the incident vertex. The traversal operation that is not directly accessible with a single pointer access is available through the opposite halfedge. For example the next halfedge around the incident source vertex for the FE-structure is opposite()->next(). The different conventions are not independent. If the convention defines the halfedge order around a facet to be clockwise, the halfedge order around the vertex will be counterclockwise, and vice versa.

The halfedge data structure is able to model orientable 2-manifolds. It is sufficient for modeling topology even in the presence of loops and multi-edges, which can occur in curved-surface environments [32]. High-level operations maintaining integrity are again Euler operators. The solid modeling system GWB [19] is based on a halfedge-data structure, though it uses an additional edge record between two opposite halfedges, which makes this access again inefficient. The Minimal Rendering Tool MRT [3] uses a halfedge data structure for polygonal surfaces.

Quad-Edge Data Structure. If we perform both halving steps for the halfedge data structure, we end up with the quad-edge data structure [12]. It provides a fully symmetric view on the primal and the dual graph, as can be seen in Figure 6. Instead of using opposite pointers, a two bit counter $r$ is used to address a slot in an edge record of four quad-edges. With an additional bit $f$ per edge for the flipped status the quad-edge data structure is able to model non-orientable 2-manifolds. 
A quad-edge data structure is defined as an edge algebra with three operations: Onext(), Rot() and Flip(). An edge is represented as a triple $(e, r, f)$ with $r \in$ $\{0,1,2,3\}$ and $f \in\{0,1\}$. $e$ is the base pointer to the quad-edge record with the four incident edges $e[0]$ to $e[3]$. The operations are implemented as follows with a calculus modulus 4 for $r$ and modulus 2 for $f$ :

$$
\begin{aligned}
\operatorname{Rot}(e, r, f) & =(e, r+1+2 f, f), \\
\operatorname{Flip}(e, r, f) & =(e, r, f+1), \\
O \operatorname{next}(e, r, f) & =\operatorname{Flip}^{f}\left(\operatorname{Rot}^{f}(e[r+f])\right) .
\end{aligned}
$$

Four different orientations of an edge are considered: two orientations from vertex to vertex and two orientations for the dual edge from facet to facet. The Rot operator rotates the edge by 90 degrees, oscillating between the primal and the dual view of the structure. For non-orientable 2-manifolds an edge can additionally be seen from above or below the surface, which is encoded in the $f$ bit. The Flip operation changes the view from above to below or vice versa. The Onext operation gives the next quad-edge in counterclockwise order around the source vertex (origin), or the next quad-edge in clockwise order if $f$ is equal to one. The values for Onext are simply stored in the record for each edge (i.e. four pointers and four times three bits for $r$ and $f$ ). The operations simplify considerably for orientable 2-manifolds. They can be further simplified if the dual graph is not necessary. This reduces to the winged-edge data structure enriched with a bit to encode orientation.

The single high-level operation that modifies a quad-edge data structure is the Splice() operation. It is its own dual. The usual Euler operators can be implemented in terms of Splice(). The quad-edge data structure provides a unified view for the primal and dual graph. This implies that vertices and facets cannot be distinguished with strong type checking at compile time. The definition used for duality implies, furthermore, that the facets must have a single connected boundary. Holes in facets are not allowed. If strong type checking is desired, the Splice () operation is needed twice, once for the primal view and once for the dual view. Splice() can also be provided for the halfedge data structure.

Comparison of Edge-Based Representations. The main differences of these edge representations are captured in Table 1 . The differences in the basic traversal capabilities are not negligible, especially when considering modern microprocessor architectures where conditional branching can be an order of magnitude slower than computing. The storage size requirements are quite similar. Our design will focus on the flexibility of trading runtime against storage costs. We are interested in the minimal and maximal configurations for the halfedge data structure and the space efficiency of the quad-edge data structure. Another issue is the preference for strong type checking at compile time. Polyhedral surfaces have different information stored in the vertices and facets, namely points and plane equations. These can be treated as duals of each other, but 


\begin{tabular}{|l||c|c|c|}
\hline \multicolumn{1}{|c||}{} & Winged-Edge & Half-Edge & Quad-Edge \\
\hline \hline Modeling space & \multicolumn{2}{|c|}{ orientable 2-manifold } & 2-manifold \\
Operations & \multicolumn{2}{|c|}{ Euler operator } & \multicolumn{1}{c|}{ Splice() } \\
Duality & at compile time with adaptor & at runtime with Rot operator \\
\hline Holes in facets & yes & yes & no \\
\hline Basic traversal & case distinction & direct access & modulus operation \\
\hline Min size per edge & $4 \mathrm{ptr}$ & $4 \mathrm{ptr}$ & $2 \mathrm{ptr}+2 \mathrm{bits}$ \\
\hline Max size per edge & $8 \mathrm{ptr}$ & $10 \mathrm{ptr}$ & $8 \mathrm{ptr}+12 \mathrm{bits}$ \\
\hline
\end{tabular}

Table 1: Comparison of the edge-based data structures.

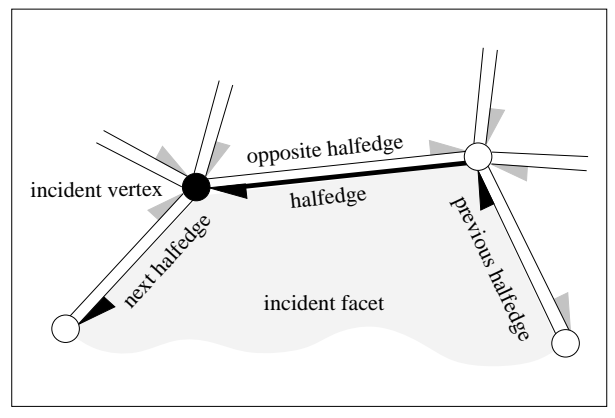

Figure 7: Halfedge data structure.

in a strongly-typed geometry kernel (like the one CGAL provides) they are different types and might even be represented differently. Additional information, like color, will finally destroy the typeless symmetry of the duality assumed by the quad-edges. We consider non-orientability as not so important since three-dimensional surfaces of solid objects are always orientable.

The choice for our design is a halfedge data structure like the FE-structure. The conventions used are depicted in Figure 7. We have next(), opposite() and prev() pointers for the halfedges. The incident vertex is the target vertex of the oriented halfedge. The incident facet is to the left of the halfedge which implies a counterclockwise ordering of the halfedges around the facet and a clockwise ordering around the vertex when seen from the outside. This complies with the right-hand rule for out-facing normals of plane equations for facets.

\section{Summary of Generic Programming, STL and It- erators}

The major design issues considered for polyhedral surfaces are genericity, flexibility, time efficiency, space efficiency and ease-of-use. Two techniques are available in $\mathrm{C}++$ for realizing generic and flexible designs: Object-oriented programming, using inheri- 
tance from base classes with virtual member functions, and generic programming, using template classes and functions.

The object-oriented programming paradigm has the advantage of defining the interface explicitly with a base class that derived implementations must follow. There are three disadvantages: It enforces tight coupling through the inheritance relationship [17], it adds additional memory to each object derived from that type (the so-called virtual function table pointer) and it adds an indirection through the virtual function table for each call to a virtual member function [18]. The latter one is of particular interest when considering runtime performance since virtual member functions can usually not be made inline and are therefore not subject to code optimization within the calling function. Modern microprocessor architectures ${ }^{4}$ can optimize at runtime. Runtime predictions are difficult, but these mechanisms are more likely to fail for virtual member functions. Such a failure will typically result in a loss in runtime of one or two orders of magnitude. Examples are low-dimensional vector arithmetic or traversals of combinatorial structures. An advantage is the dynamic type checking possibility, which gives rise to runtime flexibility, but this means that the compiler cannot provide strong type checking for these kinds of classes.

The generic programming paradigm features what is known in $\mathrm{C}++$ as templates. It provides strong type checking at compile time, does not need extra storage and allows inline member functions and code optimization [30]. One disadvantage is the lack of a formal scheme in the language for expressing the requirements of template arguments. This is left to the program documentation. It is even the case that a class may not implement all requirements for a template argument as long as these requirements are not used by the actual instantiation of the template. In particular, the implicit instantiation of a template class is only allowed to instantiate those member functions that are actually used [4]. This enables us to design template classes with optional functionality that impose additional requirements on the template arguments if and only if this functionality is used. Objects, such as vertices, edges and facets, are anticipated to be small objects with simple member functions. The space and runtime overhead introduced through virtual member functions might not be negligible. We have chosen the template technique for the design presented here, as well as in many other places in CGAL.

A good example for the generic programming paradigm is the Standard Template Library, STL [29, 23, 4, 27]. The main source of its generality and flexibility stems from the separation of concepts and models. For example, an iterator is an abstract concept defined in terms of requirements. A certain class is said to be a model of the concept if it fulfills the requirements. An iterator is a generalization of a pointer and the usual C-pointer is a model of an iterator. Iterators serve two purposes: They refer to an item and they allow the traversal over the sequence of items in a container class. Different

\footnotetext{
${ }^{4}$ pipelining, branch prediction, speculative execution and reordering, global optimizers using runtime statistics and the interplay with the cache architecture.
} 
categories are defined for iterators: input, output, forward, bidirectional and randomaccess iterators. They differ mainly in the traversal capabilities. The usual C-pointer is a random-access iterator. Generic algorithms in the STL are not written to work directly with a particular container class but on a pair of iterators. The so called range [first, beyond) of two iterators denotes the sequence of items beginning with $*$ first up to but not including *beyond. A container is supposed to provide a type which is a model of an iterator and two member functions: begin() returns the start iterator of the sequence and end () returns the iterator referring to the 'past-the-end'-position of the sequence. The generic count function in the STL could now be written as follows [27] and will work for any model of an input iterator.

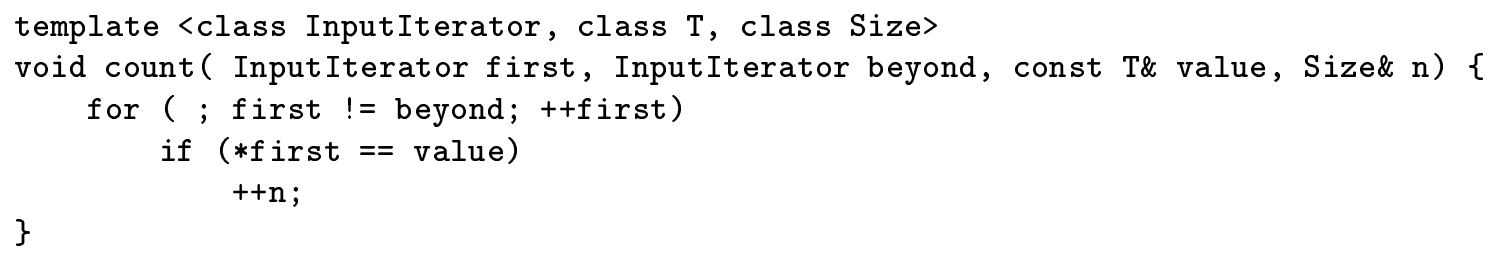

\section{Design Goals for Polyhedral Surfaces}

We adopted the generic programming paradigm for CGAL and the polyhedral surface design. We define the concept polyhedron similar to STL container classes as responsible for managing the items of a polyhedral surface and their combinatorial structure. We have identified the following design issues:

1. The edge-based data structures discussed in the previous section have a natural notion of the edges around a vertex or around a facet. It would be costly to provide iterators for these kind of circular sequences since the notion of ranges and the 'past-the-end' value do not extend naturally. We propose a concept similar to iterators - what we call circulator - for this kind of structure.

2. STL containers base their interface on iterators. For polyhedral surfaces the order of the stored items is not well-defined in certain situations, e.g. after Euler operations. Here we fall back on the concept of handles, which is the item-denoting part of iterators and ignore the traversal capabilities. In particular, any iterator and any circulator is a model for a handle.

3. The actual storage organization of the vertices, edges and facets influences the space and runtime efficiency. A doubly-connected list representation allows random insertion and removal while providing bidirectional iterators that enumerate all items. A more space efficient storage uses an STL vector which allows only the efficient removal of items at the end of the vector but provides random-access 
iterators. Other variants, like managing chunks of memory or simple allocation on the heap, could be anticipated without any iterators at all.

4. The necessary incidence information might depend on the application. The minimum needed for traversals are next() and opposite() pointers. The prev() pointer can be simulated with a search around the vertex or facet. For triangulations this is still a simple expression $(\operatorname{prev}() \equiv \operatorname{next}() \rightarrow \operatorname{next}())$ and for constant degree a constant time operation. If no information needs to be attached to vertices or facets, no storage should be allocated for them, including the referencing pointer in the edges. In its extreme the data structure reduces to an undirected graph.

5. It should be easy to add additional information to the different items, e.g. color to facets. Geometry will be attached using the same technique. Modifying one item should not hinder the re-use of the other items, for example, adding color to facets should not imply that a new vertex type must be declared.

6. The data structure should provide an easy-to-use high-level interface. This interface should protect the internal combinatorial integrity of the data structure as given in Definition 2.3. Advanced algorithms concerned with efficiency, e.g. a file format scanner, should be allowed to access the internal structure in a controlled fashion.

7. The management of connected components and containment relations, e.g. holes in facets or shells, is seen as an independent functionality with its own layer. Different solutions can be envisioned.

We concentrate on the combinatorial aspects of the polyhedral surface. Additional issues will appear when considering geometry, for example flexibility with the point type and the geometric predicates. One technique explored for this in CGAL is an extension $[5,10]$ of the traits classes known from the C++ standard library [24].

\section{Previous Work}

The Library of Efficient Datatypes and Algorithms (LEDA) [20, 21] contains no data structure tailored for three-dimensional polyhedrons, but it provides a general data structure for graphs and one for planar maps derived from graphs. Additional information can be attached either by parameterized graphs or by node arrays and edge arrays. These are associative arrays (hash tables) which allows the easy addition of information even for temporary purposes. The disadvantage of the parameterized graph is that one must always specify both parameters. The disadvantages of the node and edge arrays are the additional costs for the lookup operations and additional storage requirements. 
A more subtle disadvantage is that a reference to a node is not sufficient to retrieve its associated attributes. The array must be known too. The current size of graph nodes is equivalent to 13 pointers and for halfedges it is 11 pointers. There is no flexibility for obtaining smaller graph structures. LEDA has its own notion of iterators and a rich body of algorithms for working on graphs. At the moment it is not compliant to the STL. LEDA is very homogeneous and easy-to-use. Its own framework is generic and flexible but (currently) monolithic when combined with other libraries like the STL. It does not reach the flexibility in runtime and space efficiency tuning achieved with the approach presented in this paper.

The Minimal Rendering Tool MRT uses a halfedge data structure to represent polyhedral surfaces [3]. It is implemented as a C++ class hierarchy and provides Euler operations to maintain combinatorial integrity. The internal representation is accessible at construction time and protected thereafter. No other access is granted. It separates geometry and topology except for vertices where a point is incorporated just at the combinatorial level for efficiency reasons. Flexibility and genericity are achieved with virtual member functions for geometric properties. No flexibility is available at the topological level. Facets are responsible for storing the ring of halfedges of their boundary. Summarizing, this approach leads to larger nodes for vertices, halfedges and facets and slower functions for geometric properties than the solution we developed.

\section{Circulators}

Our new concept of circulators reflects the fact that combinatorial structures often lead to circular sequences, in contrast to the linear sequences supported with iterators and container classes in the STL. Examples of polyhedral surfaces and planar maps give rise to the circular sequences of edges around a vertex or a facet. Implementing iterators for circular sequences is possible, but not straightforward since no natural past-theend situation is available. An arbitrary sentinel in the cyclic order would break the natural symmetry in the configuration, which is in itself a bad idea, and will lead to cumbersome implementations. Another solution stores, within the iterator, a starting edge, a current edge and a kind of winding-number that is zero for the begin()-iterator and one for the past-the-end iterator ${ }^{5}$. No solution is known to us that would provide a light-weight iterator as it is supposed to be (in terms of space and efficiency). In CGAL we therefore introduced the concept of circulators, which does allow light-weight implementations. Adaptor classes will convert between iterators and circulators, thus integrating this new concept into the framework of the STL.

\footnotetext{
${ }^{5}$ This is currently implemented in CGAL as an adaptor class which provides a pair of iterators for a given circulator.
} 
Circulators share most of their requirements with iterators. Three circulator categories are defined: forward, bidirectional and random-access circulators. Given a circulator c the operation $* c$ denotes the item the circulator refers to. The operation $++c$ advances the circulator by one item and --c steps a bidirectional circulator one item backwards. For random-access circulators $c+n$ advances the circulator by $n$ where $n$ is a natural number. Two circulators can be compared for equality.

Circulators develop different notions of reachability and ranges than iterators. A circulator $\mathrm{d}$ is called reachable from $\mathrm{c}$ if $\mathrm{c}$ can be made equal to $\mathrm{d}$ with finitely many applications of the operator $++c$. Due to the circularity of the data structure this is always true if both circulators refer to items of the same data structure. In particular, $c$ is always reachable from $c$. Given two circulators $c$ and $d$, the range $[c, d$ ) denotes all items starting with $* c$ up to but not including $*$ d. So far it is the same range definition as for iterators. The Difference lies in the use of $[c, c)$ for denoting all items in the circular data structure whereas for an iterator $i$ the range $[i, i)$ denotes the empty range. As long as $c \quad !=d$ the range $[c, d)$ behaves like an iterator range and could be used in STL algorithms. It is possible to write just as simple algorithms that work with iterators as well as with circulators, including the full range definition [6, Chapter 3.7.4]. An additional test $c==$ NULL is now required that is true if and only if the data structure is empty. In this case the circulator $\mathrm{c}$ is said to have a singular value. For the complete description of the requirements for circulators please refer to [6, Chapter $3.7 .2]$.

We repeat the example for the generic count() function from above for a range of circulators. The main difference is the use of a do ... while() loop instead of a for () loop.

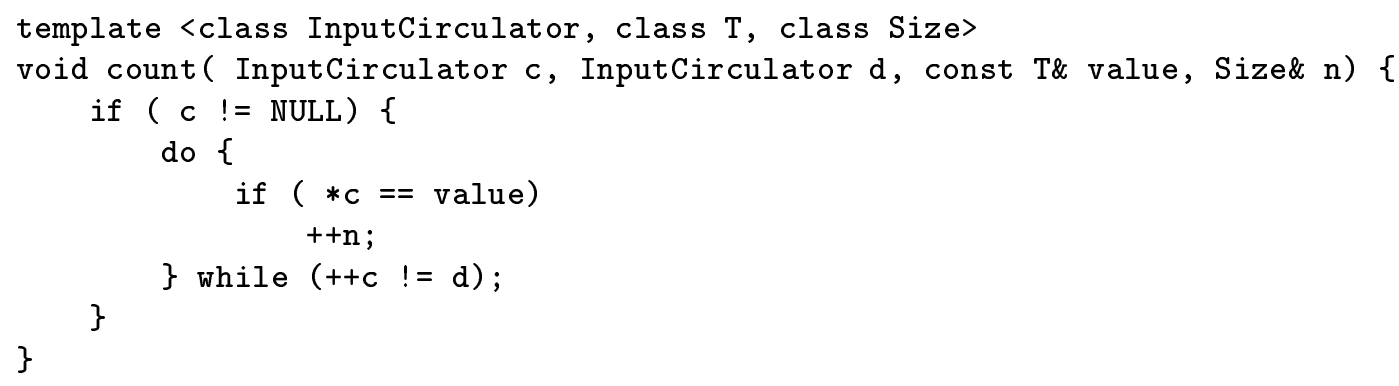

\section{Design Overview}

The global picture of the design is given in Figure 8. The design strictly separates topology and geometry. Vertices, halfedges and facets carry both kinds of information. The Halfedge_data_structure is the container managing these three items and 


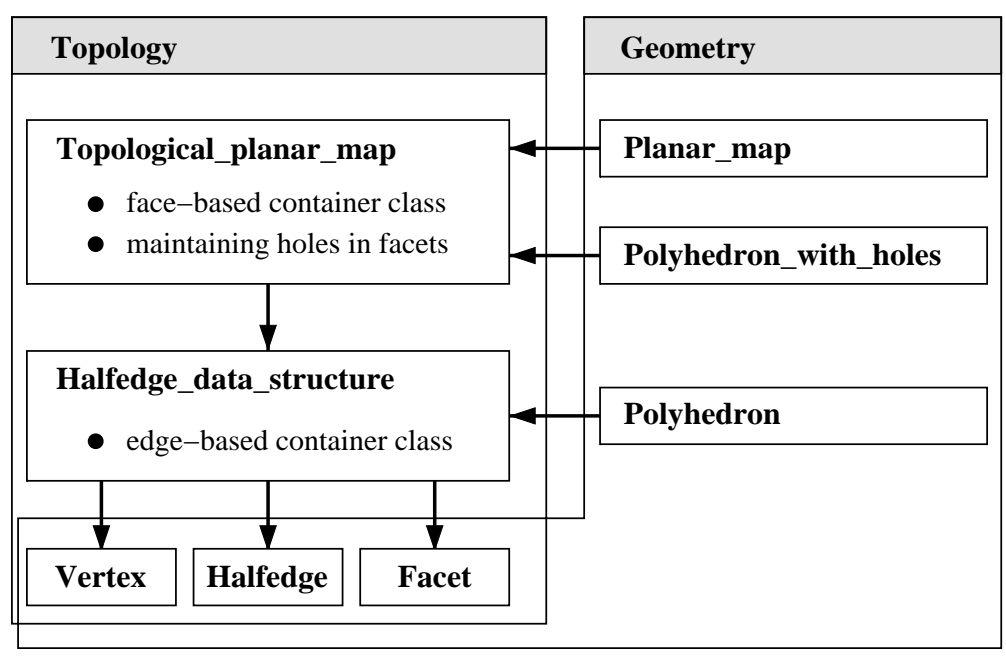

Figure 8: Design overview.

their topological relations. The Topological_planar_map is a face-based representation. It maintains holes in facets and is able to enumerate outer and inner boundaries of a facet. It uses the edge-based data structure. The Polyhedron uses the Halfedge_data_structure and adds geometric operations. It imposes further restrictions on the data structure as defined for the polyhedral complex above, for example, that an edge always has two distinct endpoints. The Planar_map and a possible Polyhedron_with_holes are based on the topological planar map since they will maintain holes in facets.

All entities in this picture are sets of requirements. Each can have multiple models. There are many different possibilities for vertices, edges and facets. Currently two different models are provided for the Halfedge_data_structure. Many combinations are possible and result in different polyhedron data structures. We make use of the implicit instantiation of template classes in $\mathrm{C}++$ as described above. The requirement sets consist of a mandatory part that every model must comply with and certain optional parts a model must only comply with if the corresponding functionality is actually used. For example, a vertex is allowed to be empty. If we want to use it for the polyhedral surface then the normal vector computation imposes the additional requirements that the vertex must contain a three-dimensional point and give access to it with the member function point ().

\section{Design of the Polyhedron}

A more refined picture of the design is shown in Figure 9. At the bottom we start with base classes for vertices, halfedges and facets. Their responsibilities are the actual storage of the incidences in terms of void-pointers, the geometry and other attributes. 


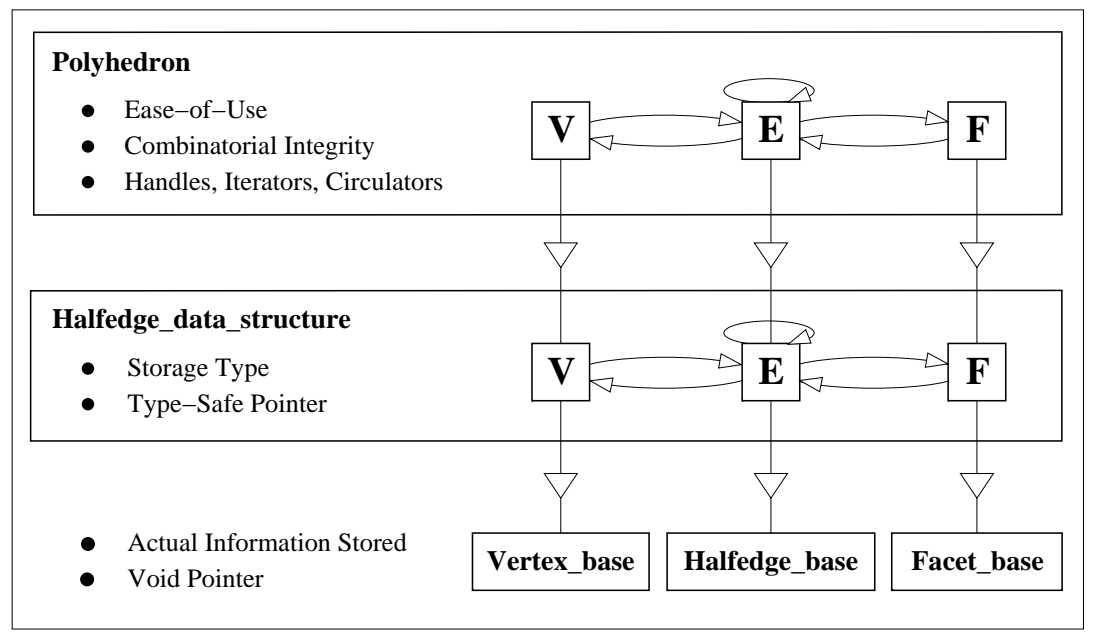

Figure 9: Responsibilities of the different layers in the polyhedron design.

Especially the storage of incidences with void-pointers allows, for example, the facet class to be exchanged without changing the halfedge class. The advantage of strong type checking will be reestablished for the void-pointers in the next layer. Implementations for vertices, edges and facets are provided that fulfill the minimal set of requirements. They can be used as base classes for own extensions. Richer implementations are provided as defaults; for polyhedrons they provide a three-dimensional point in the vertices and a plane equation in the facets.

The Halfedge_data_structure is responsible for the storage organization of the vertices, halfedges and facets. Currently implementations are provided that use a bidirectional list or an STL vector internally. The Halfedge_data_structure derives new classes for vertices, halfedges and facets. They replace the void-pointer incidence information with type-safe pointers at the interface. Additional information besides the incidence information simply stays unaffected and will be inherited from the base classes.

Different models are possible for the Halfedge_data_structure (two have already been implemented). Thus the set of requirements for the Halfedge_data_structure is kept small. What can be done if several classes need to implement similar high-level operations? The solution is the helper class Halfedge_data_structure_decorator, which is not shown in Figure 9 but would be placed at the side of the Halfedge_data_structure since it broadens that interface but does not hide it. It adds Euler operations and adaptive functionality. For example, if the prev() function is not provided for halfedges, a find_prev() function searches the previous halfedge along the facet. If the prev() function is now implemented, the find_prev() function simply calls it. This distinction can be resolved at compile time with a technique called compile-time tags, similar to iterator tags in [29]. 


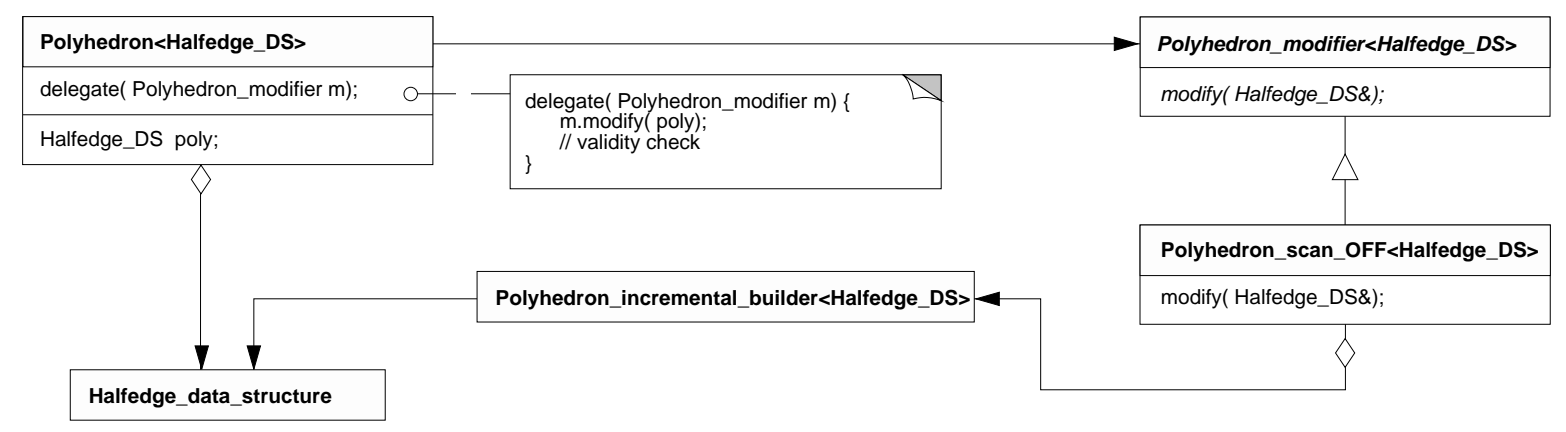

Figure 10: Class diagram of the polyhedron design illustrating the safe access to the internal representation using the strategy pattern [9].

The Polyhedron layer adds ease-of-use in terms of high-level functions, high-level concepts for accessing the items, i.e. handles, iterators and circulators (pointers are no longer visible at this interface), and the protection of the combinatorial integrity. It derives new vertices, halfedges and facets to provide the handles and hide the pointers.

Algorithms with invalid intermediate states need access to the internal representation. A protected access is granted for classes derived from Polyhedron_modifier. It follows the strategy pattern from [9], see Figure 10. The example depicted is a file format scanner Polyhedron_scan_OFF for polyhedrons stored in the Object File Format OFF [26]. The back-door is a kind of callback-function embedded in a class object. The Polyhedron accepts a modifier class with the delegate() member function and calls its modify() member function with the internal representation. The Polyhedron_scan_OFF class derives from the Polyhedron_modifier and implements the modify() function where it can access the internal representation. The trick is here that the delegate() function of the Polyhedron can verify the validity of its own internal representation after the modify() function has done its work. The Polyhedron_scan_OFF class is in charge of returning from execution only with a valid representation, even in the case of a failure. The diagram contains another helper class Polyhedron_incremental_builder, which has been designed especially for constructing polyhedral surfaces from a list of vertices and facet index lists as they usually appear in file formats. This helper class can of course fail. In this case it offers a rollback() function for deleting all elements created so far and leaves the internal representation in the (valid) state it was before starting the scan. This approach is known as transactions from databases and can be applied in other places as well. The special task Polyhedron_scan_OFF is written for enables us to implement the transaction mechanism very efficiently. In general it would be more costly. 


\section{Evaluation of the New Design}

We will illustrate in the following that our design not only meets the design goals formulated in Section 5 but that it is also still easy to use. A certain familiarity with the look-and-feel of $\mathrm{C}$ or $\mathrm{C}++$ will help in this section. The Appendix A gives a short introduction for $\mathrm{C}++$ novices.

A representation type $\mathrm{R}$ denotes geometric objects in $\mathrm{CGAL}$, such as three dimensional points and plane equations. These are used in the default implementation for the halfedge data structure. For example the following simple function foo() creates a combinatorial tetrahedron in $\mathrm{P}$ where space for points and plane equations is allocated in the vertices and facets even though it is not used here.

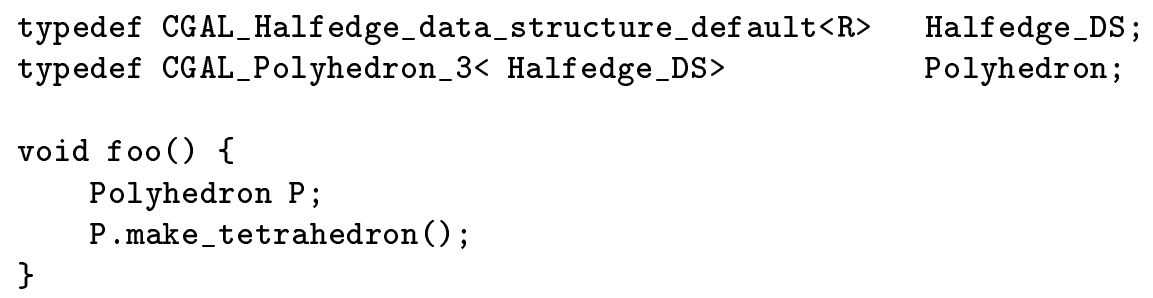

The class Polyhedron provides the claimed ease-of-use with handles, iterators, circulators and high-level operations, thus addressing design goals (1) and (2).

The default representation is actually equivalent to

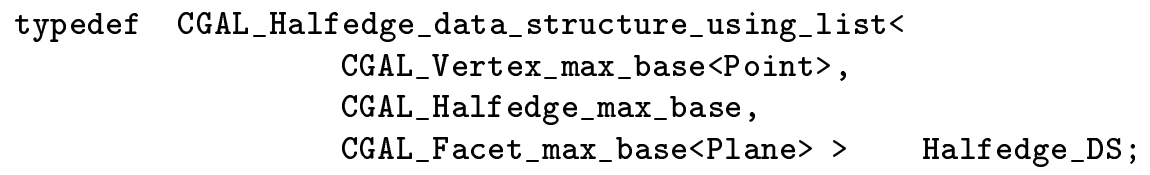

with the appropriate point and plane equation. The internal storage organization can be easily changed with the class CGAL_Halfedge_data_structure_using_vector to a vector-based one. This satisfies design goal (3). The requirements a self-written class must fulfill to be a model of a halfedge data structure are documented in [15].

Exchanging the default base classes with the minimal base classes CGAL_Vertex_min_ base, CGAL_Halfedge_min_base and CGAL_Facet_min_base yield a data structure for undirected graphs since vertices and facets are empty (besides a few compile-time tags) and the minimal halfedge base class stores only a next() and an opposite () pointer. This makes four pointers per edge. See $[15]^{6}$ for the actual short implementations

\footnotetext{
${ }^{6}$ CGAL Release 1.0 is expected to be released next year. The appendix collects the examples mentioned here.
} 
of these base classes. In analogy to the quad-edge data structure we can replace the opposite() pointer internally by a single bit knowing that opposite halfedges are always stored in consecutive places by our halfedge data structures. Knowing $\mathrm{C}$, this bit can be put into the least-significant bit of the next() pointer, which is always zero on todays systems. This yields an implementation with two pointers per edge! The easy realization of this idea in our design is shown in the manual [15]. We can add a color variable to the default facet:

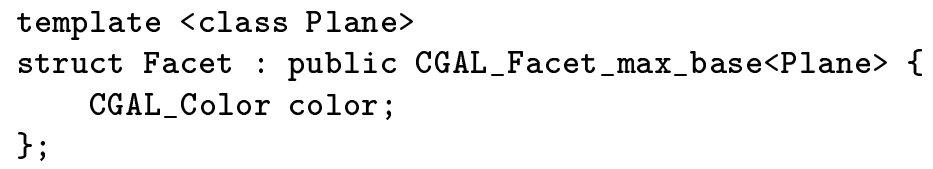

This facet can be used instead of the default facet and we have a polyhedron with colored facets. This satisfies design goal (5). Similarly easy is the implementation of incidences that lie between the minimal and default classes. We add a previous pointer to the minimal halfedge. The type Supports_halfedge_prev indicates that the class now supports a previous pointer.

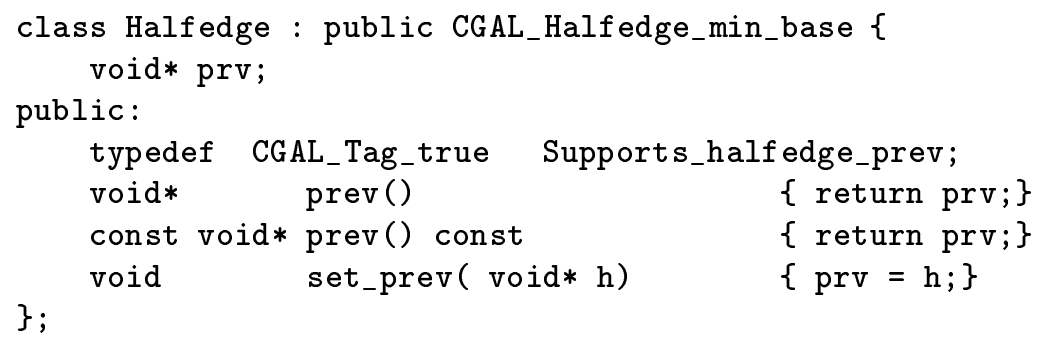

The whole spectrum of incidences is easily available in the design presented, fulfilling design goal (4). Design goals (6) and (7) are also met. The example for the easy use of the Polyhedron_modifier mechanism and the Polyhedron_incremental_builder can be found in the manual [15].

Summing up, we have met all design goals presented in Section 5 without imposing runtime and storage overhead. Predefined implementations are easy to use and different solutions within the possible flexibility can be achieved with little effort.

\section{Conclusion}

We have presented a design framework for combinatorial data structures, such as planarmaps and polyhedral surfaces. It extends to curved-surface environments and can also 
be applied to other combinatorial data structures, such as triangle-based structures for triangulations. We have identified important fundamentals for such a design: A proper definition of the modeling space, strong type checking, time and space efficiency.

The adaption of the generic programming paradigm used in the STL has led to an easy-to-use and flexible high-level interface for polyhedral surfaces featuring handles, iterators, the new concept circulators and Euler operators. The internal representation can be chosen from a wide range of different halfedge data structures exploiting many tradeoffs between time and storage efficiency, iterator categories and modifiability. Additional attributes are easy to add. Other solutions, such as dynamic type checking at runtime, generic attribute pointers or templates, can still be added within this design. The integrity of the internal representation is protected and a mechanism is available that grants safe access to it. We expect a continuation of this approach in CGAL.

Acknowledgments. I would like to thank my advisor Emo Welzl for the freedom and encouraging guidance throughout these studies, Nora Sleumer and Martin Will for reading and commenting on previous versions, and Andreas Fabri, Geert-Jan Giezeman, Stefan Schirra and Sven Schönherr for the intensive discussions on $\mathrm{C}++$ design and software engineering since we started with the CGAL kernel almost three years ago. The CGAL project has grown and I want to thank all members of the project for the inspiring environment, especially the people attending the CGAL meeting at INRIA, Sophia Antipolis, where the connections between the planar maps and the polyhedral surface design was discussed. 


\section{References}

[1] Bruce G. Baumgart. A Polyhedron Representation for Computer Vision. In National Computer Conference, pages 589-596, Anaheim, CA, 1975. AFIPS.

[2] Gavin Bell, Anthony Parisi, and Mark Pesce. VRML The Virtual Reality Modeling Language: Version 1.0 Specification. http://www.vrml.org/, May 26 1995. Third Draft.

[3] Heinzgerd Bendels, Dieter W. Fellner, and Sven Havemann. Modellierung der Grundlagen: Erweiterbare Datenstrukturen zur Modellierung und Visualisierung polygonaler Welten. In D. W. Fellner, editor, Modeling - Virtual Worlds - Distributed Graphics, pages 149-157, Bad Honnef / Bonn, 27.-28. November 1995. http://www.graphics.uni-bonn.de/CompGraph. ResearchProjects.MRT.

[4] Working Paper for Draft Proposed International Standard for Information Systems - Programming Language C++. ANSI X3, Information Processing Systems, December 1996. http: //www . maths. warwick.ac.uk/c++/pub/.

[5] CGAL Reference Manual. Part 2: Basic Library, June 1997. CGAL R0.9. http://www.cs.ruu. $\mathrm{nl} / \mathrm{CGAL}$.

[6] CGAL Reference Manual. Part 3: Support Library, June 1997. CGAL R0.9. http://www.cs . ruu.nl/CGAL.

[7] Mark de Berg, Marc van Krefeld, Mark Overmars, and Otfried Schwarzkopf. Computational Geometry: Algorithms and Applications. Springer Verlag, 1997.

[8] Andreas Fabri, Geert-Jan Giezeman, Lutz Kettner, Stefan Schirra, and Sven Schönherr. The CGAL Kernel: A Basis for Geometric Computation. In M. C. Lin and D. Manocha, editors, $A C M$ Workshop on Applied Computational Geometry, pages 191-202, Philadelphia, Pennsylvenia, May, 27-28 1996. Lecture Notes in Computer Science 1148.

[9] E. Gamma, R. Helm, R. Johnson, and J. Vlissidis. Design Patterns - Elements of Reusable Object-Oriented Software. Addison-Wesley, 1995.

[10] Geert-Jan Giezeman, Remco Veltkamp, and Wieger Wesselink. Getting Started with CGAL, June 1997. CGAL R0.9. http://www.cs.ruu.nl/CGAL.

[11] Andrew S. Glassner. Maintaining Winged-Edge Models. In James Arvo, editor, Graphics Gems II, pages 191-201. Academic Press, 1991.

[12] Leonidas Guibas and Jorge Stolfi. Primitives for the Manipulation of General Subdivisions and the Computation of Voronoi Diagrams. ACM Transaction on Graphics, 4(3):74-123, July 1985.

[13] Christoph M. Hoffmann. Geometric and Solid Modeling - An Introduction. Morgan Kaufmann, 1989.

[14] Michael Hoffmann. Line-Sweep auf einem Gitter, 1996. Diplomarbeit. Freie Univ. Berlin, Germany.

[15] Lutz Kettner. 3D-Polyhedral Surfaces. In Andreas Fabri, Stefan Schirra, and Remco Veltkamp, editors, CGAL Reference Manual. Part 2: Basic Library. 1998. CGAL R1.0. http://ww..cs . ruu.nl/CGAL. to appear.

[16] Lutz Kettner and Emo Welzl. Contour Edge Analysis for Polyhedron Projections. In Wolfgang Straßer, Reinhard Klein, and René Rau, editors, Geometric Modeling: Theory and Practice, pages -. Springer Verlag, 1997. (Proc. Int. Conf. Theory and Practice of Geometric Modeling in Blaubeuren, Germany, Oct. 1996). in press.

[17] John Lakos. Large Scale C++ Software Design. Addison-Wesley, 1996. 
[18] Stanley B. Lippman. Inside the C++ Object Model. Addison-Wesley, 1996.

[19] Martti Mäntylä. An Introduction to Solid Modeling. Computer Science Press, Rockville, Maryland, 1988.

[20] Kurt Mehlhorn and Stefan Näher. LEDA: A Platform for Combinatorial and Geometric Computing. Communications of the ACM, 38(1):96-102, January 1995.

[21] Kurt Mehlhorn, Stefan Näher, and Christian Uhrig. The LEDA User Manual, Version 3.5. LEDA Software GmbH, 66123 Saarbrücken, Germany, 1997.

[22] D. E. Muller and F. P. Preparata. Finding the Intersection of two Convex Polyhedra. Theoretical Computer Science, 7:217-236, 1978.

[23] David R. Musser and Atul Saini. STL Tutorial and Reference Guide: C++ Programming with the Standard Template Library. Addison-Wesley, 1996.

[24] Nathan C. Myers. Traits: a New and Useful Template Technique. $C++$ Report, June 1995.

[25] Mark H. Overmars. Designing the Computational Geometry Algorithms Library CGAL. In M. C. Lin and D. Manocha, editors, ACM Workshop on Applied Computational Geometry, Philadelphia, Pennsylvenia, May, 27-28 1996. Lecture Notes in Computer Science 1148.

[26] Mark Phillips. Geomview Manual: Geomview Version 1.5 for Silicon Graphics Workstations. The Geometry Center, University of Minnesota, October 1994.

[27] Silicon Graphics Computer Systems, Inc. Standard Template Library Programmer's Guide. http: //www.sgi.com/Technology/STL/, 1997.

[28] E. Steinitz and H. Rademacher. Vorlesung über die Theorie der Polyeder (unter Einschluß der Elemente der Topologie). Springer, Berlin, 1934.

[29] Alexander Stepanov and Meng Lee. The Standard Template Library. http://www.cs.rpi.edu/ imusser/doc.ps, October 1995.

[30] Bjarne Stroustrup. The C++ Programming Language. Addison-Wesley, 3rd edition, 1997.

[31] The Virtual Reality Modeling Language Specification: Version 2.0, ISO/IEC CD 14772. http: / /www.vrml.org/, August 41996.

[32] Kevin Weiler. Edge-Based Data Structures for Solid Modeling in Curved-Surface Environments. IEEE Computer Graphics and Application, 5(1):21-40, January 1985.

[33] Josie Wernicke. The Inventor Mentor: Programming Object-Oriented 3D Graphics with Open Inventor, Release 2. Addison-Wesley, 1994. 


\section{A A Simple Example Program Using Polyhedrons}

We describe here a "Hello World" program for polyhedrons in CGAL in order to make $\mathrm{C}++$ novices more comfortable with the examples provided in the paper. The CGAL library uses the prefix CGAL_ for all global names to avoid name clashes ${ }^{7}$ and the suffix _3 for three-dimensional entities.

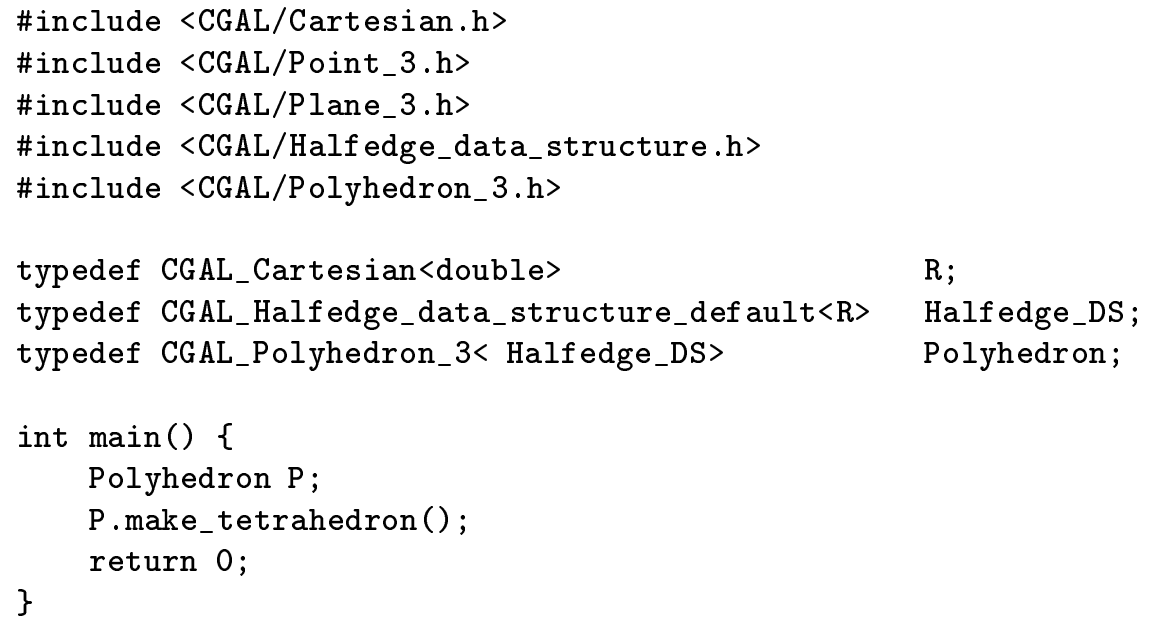

The \#include directives make the types used in the example available. We achieve genericity with template classes. Their arguments are written in angle brackets $<\ldots$. . It is convenient to use typedefs to create the nested type declarations one after the other. CGAL_Cartesian is one of the possible representation types in CGAL, parameterized with the coordinate type double. The CGAL_Halfedge_data_structure_default uses three-dimensional points for the vertices and a plane equation for the facets. Both are determined by the representation type. The CGAL_Polyhedron_3 is parameterized with the halfedge data structure. The main() function declares a variable $\mathrm{P}$ for the polyhedron and creates a combinatorial tetrahedron in $\mathrm{P}$ where space for points and plane equations is allocated in the vertices and facets even though it is not used here. The main() function exits cleanly with a return value of zero.

\section{B Program Examples Taken from the Reference Manual}

The following examples will be part of the reference manual for CGAL Release 1.0 [15], which is expected to be released next year. The examples are referenced in the paper and are collected in this appendix for the convenience of the reader.

\footnotetext{
${ }^{7}$ The name prefix will be once replaced by the namespace CGAL : : .
} 


\section{B.1 Minimal Base Classes}

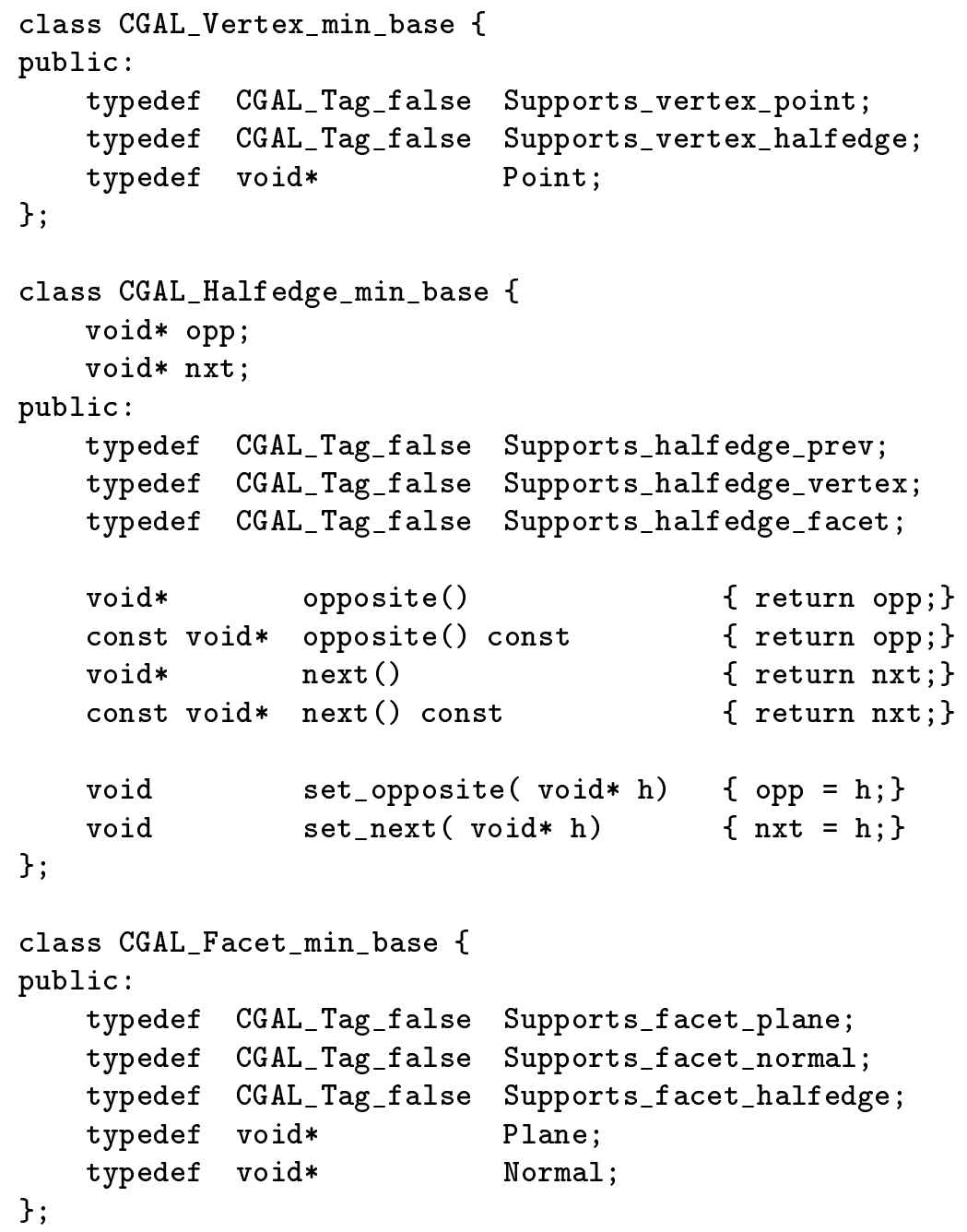

The vertex and the facet are basically empty besides the compile-time tags and dummy types for the (unused) geometry. The CGAL_Halfedge_min_base implements the interface for the functions opposite() and next() with two private member variables opp and nxt storing exactly these values.

\section{B.2 Trading Bits for Pointers}

We know from the winged-edge and the quad-edge data structure that the opposite() pointers can be replaced by a single bit if opposite halfedges are always stored in consecutive places (which they will be in the two halfedge data structures provided). This bit can be stored in the least-significant bit of the next () pointer, which is nowadays always 
zero. This yields an implementation with two pointers per edge! The following example illustrates the realization of this idea for the Halfedge_data_structure_using_vector. It implements a new halfedge class that fulfills the set of minimal requirements. It makes use of typical $\mathrm{C}$ operator notations.

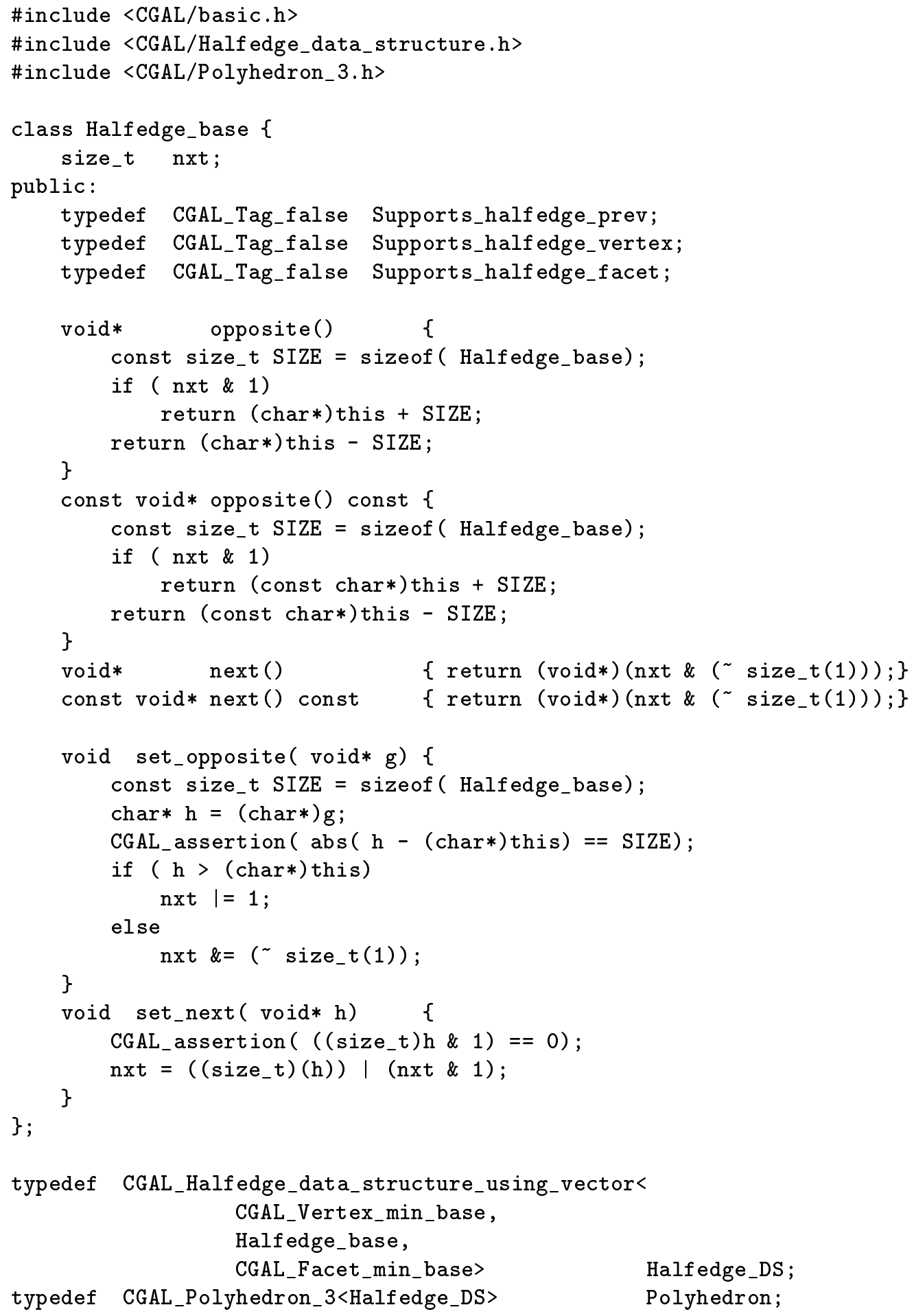




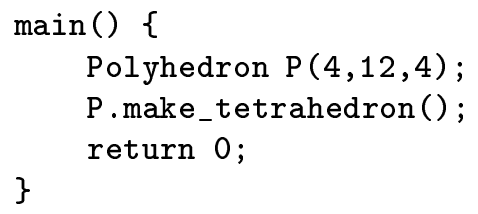

\section{B.3 The Polyhedron Modifier and the Incremental Builder}

The class Build_triangle illustrates the mechanism for accessing the internal representation of a polyhedron. It is derived from the CGAL_Polyhedron_modifier_3 class and implements the modify () member function. It can now be used as a parameter for the delegate () member function of the polyhedron. In its modify() member function the Build_triangle class makes use of the CGAL_Polyhedron_incremental_builder_3 helper class to create a triangle; first the three corners, then three indices for a single facet.

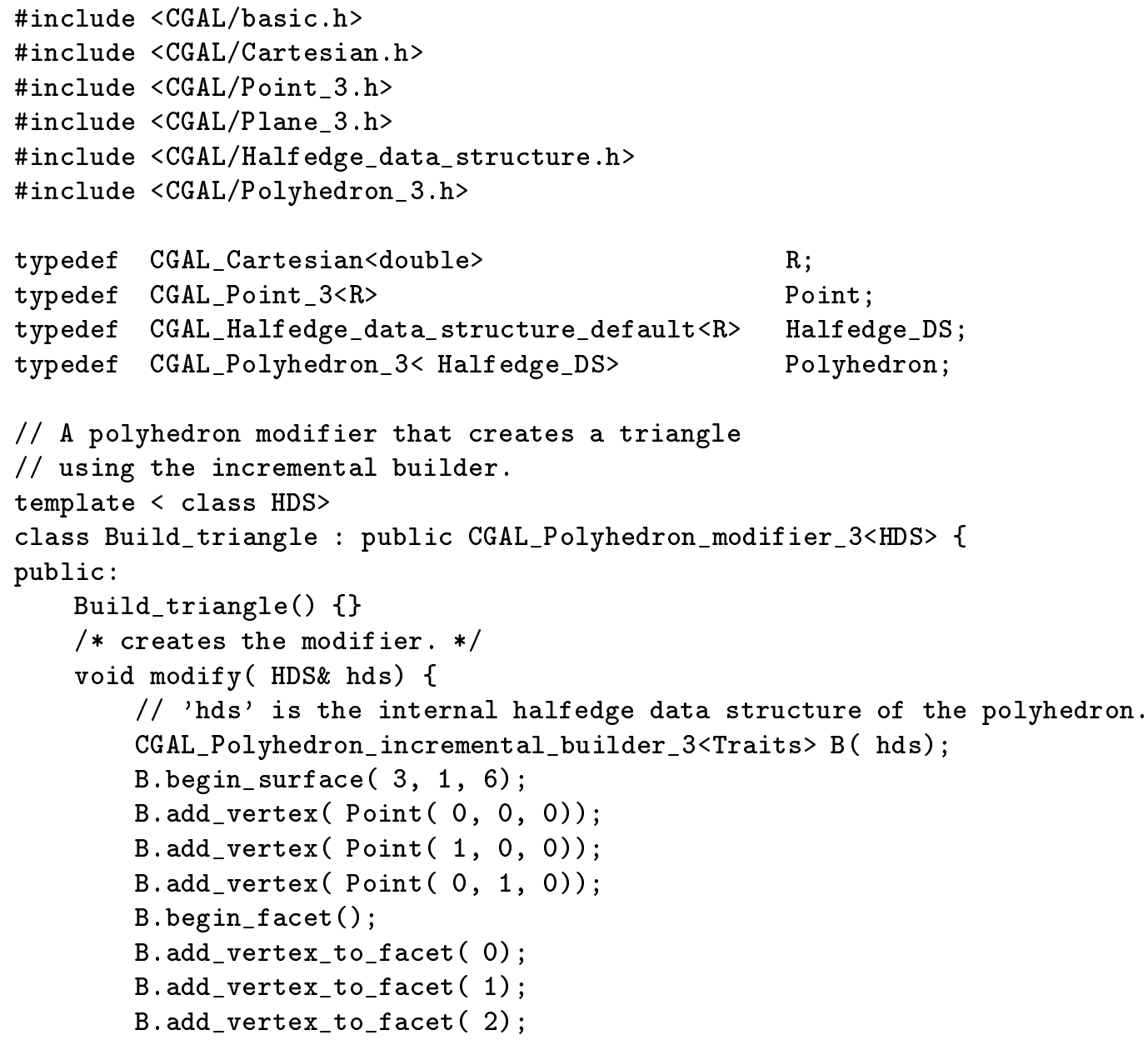




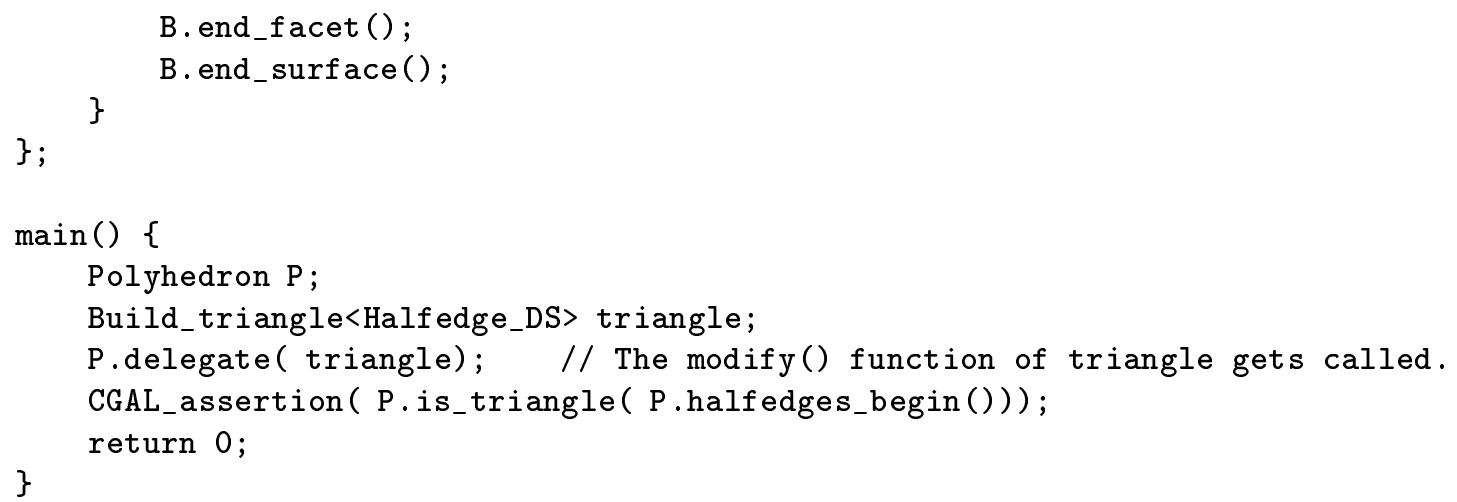

